\title{
VI-MODULES IN NON-DESCRIBING CHARACTERISTIC, PART II
}

\author{
ROHIT NAGPAL
}

\begin{abstract}
We classify all irreducible generic VI-modules in non-describing characteristic. Our result degenerates to yield a classification of irreducible generic FI-modules in arbitrary characteristic. Our result can also be viewed as a classification theorem for a natural class of representations of $\mathbf{G L}_{\infty}\left(\mathbb{F}_{q}\right)$.
\end{abstract}

\section{Contents}

1. Introduction 1

2. A cofinal sequence in the submodule-lattice of an induced module 6

3. Stabilization of the cofinal sequence, modulo torsion, in non-describing characteristic 9

4. Classification of irreducibles $\quad 12$

$\begin{array}{ll}\text { References } & 15\end{array}$

\section{INTRODUCTION}

Notation. Set $\mathbb{F}=\mathbb{F}_{q}$, and let $\mathbf{G L}_{n}$ be the $n$th general linear group over $\mathbb{F}$. Let $\mathbf{k}$ be a field in which $q$ is invertible.

A VI-module $M$ is a functor $M: \mathrm{VI} \rightarrow \operatorname{Mod}_{\mathbf{k}}$, where VI is the category of finite dimensional $\mathbb{F}$-vector spaces with injective linear maps. Since $\mathbf{G} \mathbf{L}_{n}$ acts on $M\left(\mathbb{F}^{n}\right)$, we can view $M$ as a sequence whose $n$th member is a $\mathbf{k}\left[\mathbf{G L}_{n}\right]$-module. Let $\mathcal{J}$ denote the left adjoint to the natural restriction

$$
\operatorname{Mod}_{\mathrm{VI}} \rightarrow \prod_{n \geq 0} \operatorname{Mod}_{\mathbf{k}\left[\mathbf{G L}_{n}\right]}
$$

We call VI-modules of the form $\mathcal{J}(\Theta)$ induced, and we refer to VI-modules admitting a finite filtration with induced graded pieces as semi-induced. The category $\operatorname{Mod}_{\mathrm{VI}}$ naturally contains a localizing subcategory Mod $\mathrm{VI}_{\mathrm{VI}}^{\text {tors }}$ whose members are called torsion VI-modules. We denote the Serre quotient category

$$
\operatorname{Mod}_{\mathrm{VI}} / \operatorname{Mod}_{\mathrm{VI}}^{\text {tors }}
$$

by $\operatorname{Mod}_{\mathrm{VI}}^{\text {gen }}$ and refer to its objects as generic VI-modules. See [Nag] for more on these definitions.

In non-describing characteristic, both the categories $\operatorname{Mod}_{\mathrm{VI}}^{\text {tors }}$ and $\mathrm{Mod}_{\mathrm{VI}}^{\text {gen }}$ are of Krull dimension 0 . Isomorphism classes $\operatorname{Irr}\left(\operatorname{Mod}_{\mathrm{VI}}^{\text {tors }}\right)$ of irreducible objects in $\operatorname{Mod}_{\mathrm{VI}}^{\text {tors }}$ are easy to understand and are in a natural one-to-one correspondence with

$$
\left.\bigsqcup_{n \geq 0} \operatorname{Irr}\left(\operatorname{Mod}_{\mathbf{k}[\mathbf{G L}}\right]\right)
$$

Surprisingly, the two categories $\operatorname{Mod}_{\mathrm{VI}}^{\text {tors }}$ and $\operatorname{Mod}_{\mathrm{VI}}^{\text {gen }}$ are equivalent if $\mathbf{k}$ is a field of characteristic 0; see [GLX, Theorem 3.6]. But this equivalence breaks down if $\mathbf{k}$ is not of characteristic 0 , the

2010 Mathematics Subject Classification. 13D45, 20C33, 20J05 . 
reason being - $\operatorname{Mod}_{\mathrm{VI}}^{\text {tors }}$ has finitely generated injective objects but $\operatorname{Mod}_{\mathrm{VI}}^{\text {gen }}$ doesn't. We construct all irreducibles in $\mathrm{Mod}_{\mathrm{VI}}^{\mathrm{gen}}$ in non-describing characteristic.

Theorem 1.1. Suppose $q$ is invertible in $\mathbf{k}$. Then there is a natural one-to-one correspondence

$$
\mathrm{L}: \bigsqcup_{n \geq 0} \operatorname{Irr}\left(\operatorname{Mod}_{\mathbf{k}\left[\mathbf{G L}_{n}\right]}\right) \rightarrow \operatorname{Irr}\left(\operatorname{Mod}_{\mathrm{VI}}^{\mathrm{gen}}\right) \text {. }
$$

Let $\Theta$ be an irreducible representation of $\mathbf{G L}_{n}$. The following are equivalent descriptions of $\mathrm{L}(\Theta)$ :

(a) $\mathrm{L}(\Theta)$ is the socle of $\mathcal{J}(\Theta)$ in the category of generic VI-modules.

(b) $\mathrm{L}(\Theta)$ is the generic VI-module given by the kernel of the intersections of all maps from $\mathrm{J}(\Theta)$ to VI-modules generated in degrees $<n$.

When $\mathbf{k}$ is an algebraically closed field of characteristic 0 , this description of $\mathrm{L}(\Theta)$ becomes particularly simply, and is known to experts (and also follows from the equivalence in [GLX, Theorem 3.6]). We now provide this description for completeness. Recall that the isomorphism classes of irreducible representations of $\mathbf{G L}_{n}$ are parametrized by partition valued functions - Let $\mathcal{C}_{n}$ be the isomorphism classes of cuspidal representations of $\mathbf{G L}_{n}$, and set $\mathcal{C}=\sqcup_{n \geq 1} \mathfrak{C}_{n}$. If $\rho \in \mathcal{C}_{n}$, we set $|\rho|=n$. Let $\mathcal{P}$ be the set of partitions. Given a partition $\lambda$, we set $|\lambda|=n$ if $\lambda$ is a partition of $n$. Given a function $\boldsymbol{\mu}: \mathcal{C} \rightarrow \mathcal{P}$, we set $|\boldsymbol{\mu}|=\sum_{x \in \mathcal{C}}|x||\boldsymbol{\mu}(x)|$. The isomorphism classes of irreducible representations of $\mathbf{G L}_{n}$ are in bijection with the set of functions $\boldsymbol{\mu}$ satisfying $|\boldsymbol{\mu}|=n$. We fix an irreducible representation $\Theta_{\boldsymbol{\mu}}$ corresponding to each partition function $\boldsymbol{\mu}$. Let $\iota \in \mathcal{C}_{1}$ be the trivial representation of $\mathbf{G L}_{1}$. For a partition function $\boldsymbol{\mu}$ with $\boldsymbol{\mu}(\iota)=\lambda$, we define another partition function $\boldsymbol{\mu}[n]$ by

$$
\boldsymbol{\mu}[n](\rho)= \begin{cases}\left(n-|\boldsymbol{\mu}|, \lambda_{1}, \lambda_{2}, \ldots\right) & \text { if } \rho=\iota \\ \boldsymbol{\mu}(\rho) & \text { if } \rho \neq \iota .\end{cases}
$$

This definition makes sense only if $n \geq|\boldsymbol{\mu}|+\lambda_{1}$. The following result can be easily obtained using the results in $[\mathrm{GW}]$.

Theorem $1.2([\mathrm{GW}])$. Suppose $\mathbf{k}$ is a field of characteristic 0 . Let $\boldsymbol{\mu}$ be a partition valued function. Suppose $|\boldsymbol{\mu}|=d$. Suppose $\boldsymbol{\mu}(\iota)=\lambda$. Let $\mathcal{L}\left(\Theta_{\boldsymbol{\mu}}\right)$ denote the VI-submodule of $\mathcal{J}\left(\Theta_{\boldsymbol{\mu}}\right)$ given by

$$
\mathcal{L}\left(\Theta_{\boldsymbol{\mu}}\right)\left(\mathbb{F}^{n}\right)= \begin{cases}\Theta_{\boldsymbol{\mu}[n]} & \text { if } n \geq d+\lambda_{1} \\ 0 & \text { otherwise. }\end{cases}
$$

Then the unique irreducible generic VI-module $\mathrm{L}\left(\Theta_{\boldsymbol{\mu}}\right)$ is the image of $\mathcal{L}\left(\Theta_{\boldsymbol{\mu}}\right)$ in the generic category.

Our results extend the theorem above to non-describing characteristic and also strengthen it in characteristic 0 . To be more precise, let $\mathrm{T}: \operatorname{Mod}_{\mathrm{VI}} \rightarrow \operatorname{Mod}_{\mathrm{VI}}^{\text {gen }}$ denote the localization functor, and let $\mathrm{S}: \operatorname{Mod}_{\mathrm{VI}}^{\text {gen }} \rightarrow \operatorname{Mod}_{\mathrm{VI}}$ be its right adjoint (the section functor). We have the following result.

Theorem 1.3. Suppose $q$ is invertible in $\mathbf{k}$. Let $\Theta$ be an irreducible representation of $\mathbf{G L}_{d}$. Let $\mathcal{L}(\Theta)$ denote the VI-module given by the kernel of the intersections of all maps from $\mathrm{J}(\Theta)$ to semi-induced VI-modules generated in degrees $<d$. Then we have the following:

(a) $\mathrm{S}(\mathrm{L}(\Theta))=\mathcal{L}(\Theta)$. In other words, the image of a nonzero VI-submodule $M \subset \mathcal{J}(\Theta)$ is ismorphic to $\mathrm{L}(\Theta)$ in the generic category if and only if $M \subset \mathcal{L}(\Theta)$.

(b) $\mathcal{L}(\Theta)$ is generated in degrees $\leq 2 d$. In fact, Castelnuovo-Mumford regularity of $\mathcal{L}(\Theta)$ is at most $2 d$. 
(c) There is a polynomial $P$ of degree exactly d such that

$$
\operatorname{dim}_{\mathbf{k}} \mathcal{L}(\Theta)\left(\mathbb{F}^{n}\right)=P\left(q^{n}\right) \text { for } n>2 d-2 .
$$

Moreover, if $\mathbf{k}$ is a field of characteristic 0 and $\Theta=\Theta_{\boldsymbol{\mu}}$, then the two descriptions of $\mathcal{L}\left(\Theta_{\boldsymbol{\mu}}\right)$ as in this theorem and the previous theorem agree.

Remark 1.4. We note that the category of VI-modules is locally noetherian; see [PS] or [SS5]. It follows that $\mathcal{L}(\Theta)$ is a finitely generated VI-module.

Question 1.5. Suppose $q$ is invertible in $\mathbf{k}$, and let $\mathcal{L}(\Theta)$ be as in the theorem above.

(a) What is the precise degree of generation of $\mathcal{L}(\Theta)$ as a function of $\Theta$ ? In characteristic 0 , it is easy to see that the answer to this question is $|\boldsymbol{\mu}|+\lambda_{1}$.

(b) We provide an explicit generator for the $\mathbf{G L}_{n}$ representation $\mathcal{L}(\Theta)\left(\mathbb{F}^{n}\right)$ for any $n \geq d+(1+$ $\left.q+\cdots+q^{d-1}\right)$. But we prove that $\mathcal{L}(\Theta)$ is generated in degrees $\leq d$. Can we construct an explicit generator for $\mathcal{L}(\Theta)\left(\mathbb{F}^{2 d}\right)$ ?

(c) Is it true that, for any irreducible representation $\Theta$, the $\mathbf{G L}_{n}$-representation $\mathcal{L}(\Theta)\left(\mathbb{F}^{n}\right)$ is irreducible for infinitely many $n$.

(d) Can we calculate the dimension of $\mathcal{L}(\Theta)\left(\mathbb{F}^{n}\right)$ as a function of $\Theta$ ?

(e) Can we calculate extensions $\operatorname{Ext}\left(\mathrm{L}\left(\Theta_{1}\right), \mathrm{L}\left(\Theta_{2}\right)\right)$ explicitly? Or the local cohomology groups for $\mathrm{L}(\Theta)$ explicitly?

Remark 1.6. In characteristic 0, answers to all the parts, except Part (e), of the question above can easily be spelled out. It is possible to follow arguments as in [SS1] to answer part (e) to some extent but we do not pursue it in this paper.

1.1. The main idea and a partial result in defining characteristic. Let $(P, \leq)$ be a poset. A subset $S \subset P$ is cofinal if for any $x \in P$ there is a $y \in S$ such that $x \leq y$. By the submodulelattice of a module $M$, we mean the poset of submodules of $M$ under reverse inclusion. Let $\Theta$ be an irreducible representation of $\mathbf{G L}_{d}$. We provide an explicit construction of a sequence of nonzero submodules

$$
M_{d, \Theta} \supset M_{d+1, \Theta} \supset M_{d+2, \Theta} \supset \ldots
$$

of $\mathcal{J}(\Theta)$ which, together with the 0 submodule, form a cofinal subset in the submodule lattice of the induced module $\mathcal{J}(\Theta)$. The following result does not need the non-describing characteristic assumption.

Theorem 1.7. Let $\mathbf{k}$ be a field of arbitrary characteristic. Let $\Theta$ be an irreducible representation of $\mathbf{G L}_{d}$ over $\mathbf{k}$. Suppose $M \subset \mathcal{J}(\Theta)$ is any nonzero submodule. Then $M_{n, \Theta} \subset M$ for $n$ large enough. In particular, if $M\left(\mathbb{F}^{n}\right) \neq 0$, then $M_{m, \Theta} \subset M$ for all $m \geq n$.

We then use the non-describing characteristic assumption to show that this cofinal sequence stabilizes up to torsion, that is, it stabilizes in the Serre quotient category $\operatorname{Mod}_{\mathrm{VI}}^{\mathrm{gen}}$.

Theorem 1.8. Suppose $q$ is invertible in $\mathbf{k}$. Let $\Theta$ be any representation of $\mathbf{G L}_{d}$. Then the descending chain $M_{d, \Theta} \supset M_{d+1, \Theta} \supset \ldots$ stabilizes in $\operatorname{Mod}_{\mathrm{VI}}^{\text {gen }}$. In fact, $M_{d, \Theta} / M_{n, \Theta}$ is supported in degrees $<n+q^{d(n-d)}\left(1+q+\cdots+q^{d-1}\right)$. In other words, $M_{n, \Theta}=M_{d, \Theta}$ in $\operatorname{Mod}_{\mathrm{VI}}^{\mathrm{gen}}$ for each $n \geq d$.

The two theorem above let us conclude that, in non-describing characteristic, the image of $M_{n, \Theta}$ for any $n \geq d$ in the generic category is the irreducible $\mathrm{L}(\Theta)$ from Theorem 1.1. The claim that these form a complete set of irreducibles of the generic category then follows quite formally from 
the structure theory for VI-modules developed in the first paper of this sequel. The novelty of this paper lies in the two theorems above whose proofs occupy $\S 2$ and $\S 3$ respectively.

We note that the theory of VI-modules in defining characteristic is much harder and only very little of the structure theory is known. Our Theorem 1.7 provides a hint in this direction. We pose some conjectures in equal characteristic.

Question 1.9. Suppose $\mathbf{k}=\mathbb{F}$. Let $\Theta$ be an irreducible representation of $\mathbf{G L}_{d}$.

(a) Is it true that

$$
\lim _{m \rightarrow \infty} \frac{1}{q^{d m}} \operatorname{dim}_{\mathbf{k}}\left(\frac{M_{n, \Theta}}{M_{n+1, \Theta}}\right)\left(\mathbb{F}^{m}\right)=0 ?
$$

(b) When $d=1$, is it true that $\frac{M_{d, \Theta}}{M_{n, \Theta}}$ is a polynomial growth functor? If so, what is the degree?

(c) Can we classify all finitely generated polynomial growth functors? (It is proven in [Nag] that any polynomial growth functor in non-describing characteristic is eventually constant.)

1.2. The case of FI-modules. For a partition $\mu$, define another partition $\mu[n]$ by

$$
\mu[n]=\left(n-|\mu|, \mu_{1}, \mu_{2}, \ldots\right) .
$$

In characteristic 0, Church-Ellenberg-Farb [CEF] showed that for every finitely generated FI-module $M$, there is a finite set $F$ of partitions such that

$$
M_{n}=\bigoplus_{\mu \in F} \mathbf{M}_{\mu[n]}
$$

for large enough $n$, where $\mathbf{M}_{\lambda}$ denote the Specht module corresponding to the partition $\lambda$. SamSnowden [SS1] showed a stronger result that

$$
\mathcal{L}\left(M_{\mu}\right):=\bigoplus_{n \geq|\mu|+\mu_{1}} M_{\mu[n]}
$$

is an irreducible in the category of generic FI-modules, and that all irreducibles in this category are of this form. This establishes a natural one-to-one correspondence between $\operatorname{Irr}\left(\operatorname{Mod}_{\mathrm{FI}}^{\mathrm{gen}}\right)$ and $\bigsqcup_{n \geq 0} \operatorname{Irr}\left(\operatorname{Mod}_{\mathbf{k}\left[S_{n}\right]}\right)$. Church-Ellenberg-Farb's result holds in positive characteristic if we pass to the Grothendieck group and allow negative coefficients; see [Har2]. But away from characteristic 0, a classification of irreducibles for the category of generic FI-modules was not known previously. Our method for classification of irreducibles for VI-modules degenerates to yield the following result.

Theorem 1.10. Suppose $\mathbf{k}$ is an arbitrary field. Then there is a natural one-to-one correspondence between $\operatorname{Irr}\left(\operatorname{Mod}_{\mathrm{FI}}^{\text {gen }}\right)$ and $\bigsqcup_{n \geq 0} \operatorname{Irr}\left(\operatorname{Mod}_{\mathbf{k}\left[S_{n}\right]}\right)$.

Our explicit description of the irreducibles in the generic category and Theorem 1.1 and Theorem 1.3 also degenerate to yield analogous results for FI-modules in arbitrary characteristic.

Remark 1.11. We do not provide separate proofs in the case of FI-modules as they can easily be obtained by setting $q=1$ in our proofs for VI-modules. We also note that Theorem 1.8 is trivial in the case of FI-modules but is one of the main technical results in this paper. Moreover, our argument for Theorem 1.7 can be thought of as a GL version of some of the combinatorial results in [CE].

The analogue of Question 1.5, away from characteristic 0, is completely open for FI-modules as well, except for Part (b) which has no content as the two functions become equal when we plug in $q=1$. However, in characteristic 0, answer to this question and all the results in this paper are known for FI-module; see [SS1]. 
1.3. Relations to $\mathbf{G L}_{\infty}$ representations and Deligne categories. The natural inclusion $\mathbb{F}^{n} \rightarrow$ $\mathbb{F}^{n+1}$ of vector spaces induces a natural inclusion $\mathbf{G L}_{n} \rightarrow \mathbf{G L}_{n+1}$ of groups. By $\mathbb{F}^{\infty}$ and $\mathbf{G L}_{\infty}$, we denote the direct limits given by these inclusions. Let $P_{n}$ denote the subgroup of $\mathbf{G L}_{\infty}$ consisting of elements that fix $\mathbb{F}^{n} \subset \mathbb{F}^{\infty}$ pointwise. We call a $\mathbf{k}\left[\mathbf{G L}_{\infty}\right]$-module $M$ admissible if for each $x \in M$ there exists an $n$ such that every $\sigma \in P_{n}$ fixes $x$. There is a natural equivalence of categories between $\operatorname{Mod}_{\mathrm{VI}}^{\mathrm{gen}}$ and the category $\operatorname{Mod}_{\mathbf{k}\left[\mathbf{G} \mathbf{L}_{\infty}\right]}^{\mathrm{adm}}$ of admissible $\mathbf{k}\left[\mathbf{G L}_{\infty}\right]$-modules. To see this, note that we have two functors

$$
\begin{aligned}
& \Psi: \operatorname{Mod}_{\mathrm{VI}} \rightarrow \operatorname{Mod}_{\mathbf{k}\left[\mathbf{G} \mathbf{L}_{\infty}\right]}^{\mathrm{adm}} \\
& \Phi: \operatorname{Mod}_{\mathbf{k}\left[\mathbf{G} \mathbf{L}_{\infty}\right]}^{\mathrm{adm}} \rightarrow \operatorname{Mod}_{\mathrm{VI}}
\end{aligned}
$$

given by

$$
\begin{aligned}
& \Psi(M)=\lim _{n} M\left(\mathbb{F}^{n}\right) \\
& \Phi(M)\left(\mathbb{F}^{n}\right)=M^{P_{n}} .
\end{aligned}
$$

It is an easy verification that $\Psi$ factors through $\operatorname{Mod}_{\mathrm{VI}}^{\text {gen }}$ and induces an equivalence $\Psi^{\prime}: \operatorname{Mod}_{\mathrm{VI}}^{\text {gen }} \rightarrow$ $\operatorname{Mod}_{\mathbf{k}\left[\mathbf{G L}_{\infty}\right]}^{\mathrm{adm}}$ where the inverse is obtained by composing $\Phi$ with the localization functor $\mathrm{T}$. Thus the following result is a corollary of Theorem 1.1 and Theorem 1.3:

Theorem 1.12. Suppose $q$ is invertible in $\mathbf{k}$. Then we have a one-to-one correspondence

$$
\bigsqcup_{n \geq 0} \operatorname{Irr}\left(\operatorname{Mod}_{\mathbf{k}\left[\mathbf{G L}_{n}\right]}\right) \rightarrow \operatorname{Mod}_{\mathbf{k}\left[\mathbf{G} \mathbf{L}_{\infty}\right]}^{\mathrm{adm}}
$$

given by

$$
\Theta \mapsto \lim _{n} \mathcal{L}(\Theta)\left(\mathbb{F}^{n}\right) .
$$

Moreover, we have $\Phi \circ \Psi^{\prime}(\mathrm{L}(\Theta))=\mathcal{L}(\Theta)$.

Remark 1.13. We do not talk about $\mathbf{G L}_{\infty}$-perspective for the rest of the paper as our results follow from the corresponding results on VI-modules and the equivalence of category mentioned above. Our method also yield an analogues result for the infinite symmetric group.

Deligne [Del] constructed families of rigid symmetric Karoubian tensor categories $\underline{\operatorname{Rep}}\left(S_{t}\right)$ for $t \in \mathbf{C}$. These categories interpolate $\operatorname{Rep}\left(S_{n}\right)$ and can be thought of as the representation theory of the symmetric group in complex dimension $t$. Deligne and Milne [DM] constructed the $\mathbf{G L}_{\infty}$ version $\operatorname{Rep}\left(\mathbf{G L}_{t}\right)$ of these interpolation categories. These categories are not abelian when $t$ is an integer. Comes and Ostrik [CO] constructed the abelian envelope $\operatorname{Rep}^{\mathrm{ab}}\left(S_{t}\right)$ of $\operatorname{Rep}\left(S_{t}\right)$. Karoubian and abelian versions of Deligne categories have been constructed for several other sequences of groups, for example, $\mathbf{G L}(m \mid n)$ [EHS]. Deligne's construction is not very well-behaved away from characteristic 0 in the sense that it does not capture enough of the modular representation theory of the symmetric groups. Harman [Har1] constructed $\underline{\operatorname{Rep}}_{\mathbf{k}}\left(S_{t}\right)$ for $t \in \mathbf{Z}_{p}$ over a field $\mathbf{k}$ of characteristic $p$, resolving a conjecture of Deligne from one of his letter to Ostrik. This category captures more refined modular representation theory of symmetric groups and can be thought of as "modular representation theory of symmetric groups in $p$-adic dimension". At least in characteristic 0 , a relation between $\operatorname{Rep}\left(S_{\infty}\right)$ and $\underline{\operatorname{Rep}}^{\mathrm{ab}}\left(S_{t}\right)$ was provided in $[\mathrm{BEH}]$ where it was proven that there is an exact symmtric monoidal faithful functor

$$
\operatorname{Rep}{ }^{\mathrm{adm}}\left(S_{\infty}\right) \rightarrow \underline{\operatorname{Rep}}^{\mathrm{ab}}\left(S_{t}\right)
$$


Question 1.14. Can we construct a Deligne category for modular representation theory of general linear group in p-adic rank, at least in non-describing characteristic? If so, do we have an exact symmetric monoidal functor from $\operatorname{Mod}_{\mathbf{k}\left[\mathbf{G L}_{\infty}\right]}^{\mathrm{adm}}$ to this Deligne category?

Acknowledgements. We thank Inna Entova-Aizenbud and Steven V Sam for discussions on the $\mathbf{G L}_{\infty}$-perspective which is outlined in $\S 1.3$.

\section{A cofinal Sequence in the Submodule-lattice of an induced module}

Let $\Theta$ be an irreducible representation of $\mathbf{G L}_{d}$. Our aim in this subsection is to show that there is a natural sequence of nonzero submodules of $\mathcal{J}(\Theta)$

$$
M_{d, \Theta} \supset M_{d+1, \Theta} \supset M_{d+2, \Theta} \supset \ldots
$$

which, together with the 0 submodule, form a cofinal subset in the submodule lattice of the induced module $\mathcal{J}(\Theta)$. This result does not need non-describing characteristic assumption. In the next section, we shall use non-describing characteristic assumption to show that this sequence stabilizes up to torsion, that is, it stabilizes in the Serre quotient category Mod $\operatorname{VI}_{\mathrm{VI}}^{\text {gen }}$.

We recall that $\mathcal{J}(\Theta)=\mathbf{k}\left[\operatorname{Hom}_{\mathrm{VI}}\left(\mathbb{F}^{d},-\right)\right] \otimes_{\mathbf{G L}_{d}} \Theta$. In particular, any element of $\mathcal{J}(\Theta)(X)$ can be written as a k-linear combination of elements of the form $[f] \otimes \theta$ where $f: \mathbb{F}^{d} \rightarrow X$ is an $\mathbb{F}$-linear injection and $\theta \in \Theta$. When $\Theta$ is the regular representation of $\mathbf{G L}_{d}$ then we denote $\mathcal{J}(\Theta)$ by simply $\mathcal{J}(d)$. In other words, we have $\mathcal{J}(d)=\mathbf{k}\left[\operatorname{Hom}_{\mathrm{VI}}\left(\mathbb{F}^{d},-\right)\right]$. To be able to define the submodule $M_{n, \Theta}$ we need some preliminaries:

Fix a morphism $f: \mathbb{F}^{d} \rightarrow X$ in VI. In other words, $f$ is an $\mathbb{F}$-linear injective map from $\mathbb{F}^{d}$ to a vector space $X$. Fix an ordered basis $\left(\mathcal{B}_{f}, \prec\right)$ of the image $\operatorname{im}(f)$ of $f$. Denote by $\mathcal{V}_{f}$ the set of triples $\left(\alpha, C, C^{\prime}\right)$ satisfying the following

(a) $\alpha \in \mathcal{B}_{f}$

(b) $C$ is a complement of the line $\mathbb{F} \alpha$ in $\operatorname{im}(f)$ satisfying $\left(\mathcal{B}_{f}\right)_{\prec \alpha} \subset C$, and

(c) $C^{\prime}$ is a complement of $W$ in $V$.

For $v \in \mathcal{V}_{f}$, we denote the corresponding triple by $\left(\alpha_{v}, C_{v}, C_{v}^{\prime}\right)$. Let $\mathcal{E}_{f}:=\sum_{v \in \mathcal{V}_{f}} \mathbb{F} e_{v}$ be the vector space freely generated by $\mathcal{V}_{f}$.

For $v \in \mathcal{V}_{f}$, let $\sigma_{v} \in \mathbf{G L}\left(X+\mathcal{E}_{f}\right)$ be the element that takes $\alpha_{v}$ to $e_{v}$ and fixes the following pointwise: $\alpha_{w}-e_{w}$ for each $w \in \mathcal{V}_{f}, C_{v}$ and $C_{v}^{\prime}$. Let $<$ be a linear order on $\mathcal{V}_{f}$ (which, for now, is independent of the order on $\left.\mathcal{B}_{f}\right)$. For a subset $S$ of $\mathcal{V}_{f}$, let $L_{S}^{<}$be the descending product $\prod_{v \in S}(\mathrm{id}-$ $\sigma_{v}$ ). This implies that if $S^{\prime}$ is an initial segment of $S$ then $L_{S}^{<}=L_{S \backslash S^{\prime}}^{<} L_{S^{\prime}}^{<}$. We will suppress the superscript ' $<$ ' when the order is implicit. Note here that, given a VI-module $M$ over $\mathbf{k}, L_{S}$ induces a k-endomorphism on $M\left(X+\varepsilon_{f}\right)$ which is functorial in $M$.

Given a morphism $g: Y \rightarrow X$ and an object $E$ in VI, we denote the direct sum of $g$ and the unique morphism $0 \rightarrow E$ by $g^{E}$. Thus $g^{E}$ is a morphism from $Y$ to $X+E$ whose image is contained in $X$. Let $g: Y \rightarrow X$ be a VI-morphism, and let $w \in \mathcal{V}_{f}$. By $\left\langle g, \alpha_{w}\right\rangle_{w}: Y \rightarrow \mathbb{F}$ we denote the unique $\mathbb{F}$-linear map such that for each $y \in Y, g(y)$ can be written as $\left\langle g(y), \alpha_{w}\right\rangle_{w} \alpha_{w}+c$ for some $c \in C_{w}+C_{w}^{\prime}$.

Lemma 2.1. Let $g: Y \rightarrow X$ be a VI-morphism. Let $S$ be a subset of $\mathcal{V}_{f}$. Then we have

$$
\left(\prod_{w \in S} \sigma_{w}\right) g^{\varepsilon_{f}}=g^{\varepsilon_{f}}+\sum_{w \in S}\left\langle g, \alpha_{w}\right\rangle_{w}\left(e_{w}-\alpha_{w}\right)
$$

where the product is descending. 
Proof. We prove the result by induction on the size of $S$. Let $y \in Y$. Then $g^{\varepsilon_{f}}(y)=g(y)=$ $\left\langle g(y), \alpha_{w}\right\rangle_{w} \alpha_{w}+c$ where $c \in C_{w}+C_{w}^{\prime}$. This implies that

$$
\sigma_{w} g^{\varepsilon_{f}}(y)=\left\langle g(y), \alpha_{w}\right\rangle_{w} e_{w}+c=\left\langle g(y), \alpha_{w}\right\rangle_{w}\left(e_{w}-\alpha_{w}\right)+g^{\varepsilon_{f}}(y) .
$$

Thus we have $\sigma_{w} g^{\mathcal{E}_{f}}=\left\langle g, \alpha_{w}\right\rangle_{w}\left(e_{w}-\alpha_{w}\right)+g^{\varepsilon_{f}}$ proving the result for $n=1$. The general result follows from it because $\sigma_{w^{\prime}}$ fixes $\left(e_{w}-\alpha_{w}\right)$ for each $w^{\prime} \in S$.

Lemma 2.2. Let $g: Y \rightarrow X$ be a morphisms in VI. Let $<_{1},<_{2}$ be two linear orders on $\mathcal{V}_{f}$ and $S$ be a subset of $\mathcal{V}_{f}$. Then $L_{S}^{<1}\left(\left[g^{\varepsilon_{f}}\right]\right)=L_{S}^{<2}\left(\left[g^{\varepsilon_{f}}\right]\right)$.

Proof. This is immediate from Lemma 2.1.

We now define $M_{n, \Theta}$ for an arbitrary representation $\Theta$ of $\mathbf{G L}_{d}$. Let $f_{n}: \mathbb{F}^{d} \rightarrow \mathbb{F}^{n}$ be the natural inclusion - the map that takes the standard basis of $\mathbb{F}^{d}$ to an initial segment of the standard basis of $\mathbb{F}^{n}$. We assume that $\left(\mathcal{B}_{F_{n}}, \prec\right)$ is this initial segment, and define $\mathcal{V}_{f_{n}}, \mathcal{E}_{f_{n}}, L_{\mathcal{V}_{f_{n}}}$ as in the previous subsection. We define $M_{n, \Theta}$ to be the VI-submodule of $\mathcal{J}(\Theta)$ generated by elements of the form $L_{\mathcal{V}_{f_{n}}}\left(\left[f_{n}^{\mathcal{E}_{f_{n}}}\right] \otimes \theta\right)$ where $\theta \in \Theta$. Note here that that $L_{\mathcal{V}_{f_{n}}}\left(\left[f_{n}^{\mathcal{E}_{f_{n}}}\right] \otimes \theta\right)=L_{\mathcal{V}_{f_{n}}}\left(\left[f_{n}^{\mathcal{E}_{f_{n}}}\right]\right) \otimes \theta$.

Proposition 2.3. We have $M_{n, \Theta} \supset M_{n+1, \Theta}$.

The proposition above follows immediate from this more general lemma.

Lemma 2.4. Suppose $\operatorname{dim}_{\mathbb{F}} Y \geq \operatorname{dim}_{\mathbb{F}} X$. Let $f: \mathbb{F}^{d} \rightarrow X, g: \mathbb{F}^{d} \rightarrow Y$ be morphisms in VI. If there exists a VI-morphism $h: X \rightarrow Y$ that takes $\mathcal{B}_{f}$ to $\mathcal{B}_{g}$ in an order preserving way and satisfies $g=h \circ f$, then $L_{\mathcal{V}_{f}}\left(\left[f^{\mathcal{E}_{f}}\right]\right)$ generate $L_{\mathcal{V}_{g}}\left(\left[g^{\mathcal{E}_{g}}\right]\right)$ in $\mathcal{J}(d)$.

Proof. Let $K$ be a complement of $\operatorname{im}(h)$ in $Y$. Let $\varphi: \mathcal{V}_{f} \rightarrow \mathcal{V}_{g}$ be the injective map taking $\left(\alpha, C, C^{\prime}\right)$ to $\left(h(\alpha), h(C), h\left(C^{\prime}\right)+K\right)$. Fix a linear order $<_{1}$ on $\mathcal{V}_{f}$ and let $<_{2}$ be a linear order on $\mathcal{V}_{g}$ such that $\varphi$ is order preserving and $\operatorname{im}(\varphi)$ is an initial segment. Let $\widetilde{\varphi}: \mathcal{E}_{f} \rightarrow \mathcal{E}_{g}$ be the natural injection induced by $\varphi$, that is, we have $\widetilde{\varphi}\left(e_{v}\right)=e_{\varphi(v)}$. Denote the direct sum map $(h+\widetilde{\varphi}):\left(X+\mathcal{E}_{f}\right) \rightarrow\left(Y+\mathcal{E}_{g}\right)$ by $\widetilde{h}$. We claim that $\widetilde{h} \circ \sigma_{v}=\sigma_{\varphi(v)} \circ \widetilde{h}$ as a linear map from $\left(X+\varepsilon_{f}\right) \rightarrow\left(Y+\varepsilon_{g}\right)$. It suffices to check that $\widetilde{h} \circ \sigma_{v}(t)=\sigma_{\varphi(v)} \circ \widetilde{h}(t)$ for $t$ in a spanning set for $X+\mathcal{E}_{f}=\mathbb{F} \alpha_{v}+C_{v}+C_{v}^{\prime}+\varepsilon_{f}$. First suppose $t=\alpha_{v}$. Then we have

$$
\widetilde{h} \circ \sigma_{v}\left(\alpha_{v}\right)=\widetilde{h}\left(e_{v}\right)=\widetilde{\varphi}\left(e_{v}\right)=e_{\varphi(v)}=\sigma_{\varphi(v)}\left(\alpha_{\varphi(v)}\right)=\sigma_{\varphi(v)}\left(h\left(\alpha_{v}\right)\right)=\sigma_{\varphi(v)} \circ \widetilde{h}\left(\alpha_{v}\right) .
$$

Now suppose $t \in C_{v}+C_{v}^{\prime}$. Then we have

$$
\widetilde{h} \circ \sigma_{v}(t)=\widetilde{h}(t)=h(t)=\sigma_{\varphi(v)}(h(t))=\sigma_{\varphi(v)} \circ \widetilde{h}(t) .
$$

Finally, suppose $t=\alpha_{w}-e_{w}$ for some $w \in \mathcal{E}_{f}$. Then we have

$\widetilde{h} \circ \sigma_{v}\left(\alpha_{w}-e_{w}\right)=\widetilde{h}\left(\alpha_{w}-e_{w}\right)=h\left(\alpha_{w}\right)-\widetilde{\varphi}\left(e_{w}\right)=\alpha_{\varphi(w)}-e_{\varphi(w)}=\sigma_{\varphi(v)}\left(\alpha_{\varphi(w)}-e_{\varphi(w)}\right)=\sigma_{\varphi(v)} \circ \widetilde{h}\left(\alpha_{w}-e_{w}\right)$.

We have proven that $\widetilde{h} \circ \sigma_{v}(t)=\sigma_{\varphi(v)} \circ \widetilde{h}(t)$ holds for $t$ in a spanning set for $X+\mathcal{E}_{f}$, and so the claim holds.

Now note that $\widetilde{h} \circ f^{\mathcal{E}_{f}}=(h \circ f)^{\mathcal{E}_{g}}=g^{\mathcal{E}_{g}}$. Thus the claim in the paragraph above implies that $\widetilde{h}_{\star}\left(L_{\mathcal{V}_{f}}^{<1}\left(\left[f^{\mathcal{E}_{f}}\right]\right)\right)=L_{\operatorname{im}(\varphi)}^{<2}\left(\left[g^{\mathcal{E}_{g}}\right]\right)$. This shows that $L_{\mathcal{V}_{f}}^{<1}\left(\left[f^{\mathcal{E}_{f}}\right]\right)$ generates $L_{\operatorname{im}(\varphi)}^{<2}\left(\left[g^{\mathcal{E}_{g}}\right]\right)$. Since $\operatorname{im}(\varphi)$ is an initial segment of $\mathcal{V}_{g}$, we have $L_{\mathcal{V}_{g}}^{<2}\left(\left[g^{\mathcal{E}_{g}}\right]\right)=L_{\mathcal{V}_{g} \backslash \operatorname{im}(\varphi)}^{<2} L_{\operatorname{im}(\varphi)}^{<2}\left(\left[g^{\mathcal{E}_{g}}\right]\right)$ which shows that $L_{\operatorname{im}(\varphi)}^{<2}$ generates $L_{\mathcal{V}_{g}}^{<2}\left(\left[g^{\mathcal{E}_{g}}\right]\right)$. Thus $L_{\mathcal{V}_{f}}^{<1}\left(\left[f^{\mathcal{E}_{f}}\right]\right)$ generates $L_{\mathcal{V}_{g}}^{<2}\left(\left[g^{\mathcal{E}_{g}}\right]\right)$. This is independent of the orders $<_{1},<_{2}$ by Lemma 2.2, completing the proof.

We now recall and prove the following theorem from the introduction (copy of Theorem 1.7). 
Theorem 2.5. Suppose $\mathbf{k}$ is an arbitrary field. Let $\Theta$ be an irreducible representation of $\mathbf{G L}_{d}$. Suppose $M \subset \mathcal{J}(\Theta)$ is any nonzero submodule. Then $M_{n, \Theta} \subset M$ for $n$ large enough. In particular, if $M\left(\mathbb{F}^{n}\right) \neq 0$, then $M_{m, \Theta} \subset M$ for all $m \geq n$.

We need a few lemmas.

Lemma 2.6. Let $f, g: \mathbb{F}^{d} \rightarrow X$ be VI-morphisms. Suppose $\theta \in \Theta$ is nonzero. Then $L_{\mathcal{V}_{f}}\left(\left[g^{\varepsilon_{f}}\right] \otimes \theta\right)=0$ in $\mathcal{J}(\Theta)$ if and only if $\operatorname{im}(g) \neq \operatorname{im}(f)$. In particular, $M_{n, \Theta} \neq 0$ if $n \geq d$.

Proof. First suppose $W^{\prime}:=\operatorname{im}(g) \neq W:=\operatorname{im}(f)$. Since $W^{\prime} \neq W$ and $\operatorname{dim}_{\mathbb{F}} W=\operatorname{dim}_{\mathbb{F}} W^{\prime}$, there exists a $v \in \mathcal{V}_{f}$ such that $\alpha_{v} \notin W^{\prime}$ and $W^{\prime} \subset C_{v}+C_{v}^{\prime}$. It suffices to show that (id $-\sigma_{v}$ ) kills the element $\left(\prod_{w \in S} \sigma_{w}\right)\left[g^{\mathcal{E}_{f}}\right]$ for any finite subset $S$ of the initial segment $\left(\mathcal{V}_{f}\right)_{<v}$. By Lemma 2.1, we have $\left(\prod_{w \in S} \sigma_{w}\right) g^{\mathcal{E}_{f}}=g^{\mathcal{E}_{f}}+\sum_{w \in S}\left\langle g, \alpha_{w}\right\rangle_{w}\left(e_{w}-\alpha_{w}\right)$. By definition, $\sigma_{v}$ fixes each $\left(e_{w}-\alpha_{w}\right)$. Moreover, since the image of $g^{\mathcal{E}_{f}}$ is contained in $C_{v} \oplus C_{v}^{\prime} \subset V \oplus \mathcal{E}_{f}$, we see that $\sigma_{v}$ fixes $g^{\mathcal{E}_{f}}$ as well. Thus $\sigma_{v}$ fixes $\left(\prod_{w \in S} \sigma_{w}\right) g^{\mathcal{E}_{f}}$, as desired.

Next suppose $\operatorname{im}(g)=\operatorname{im}(f)=W$. For a subset $S$ of $\mathcal{V}_{f}$, set $g_{S}:=g^{\mathcal{E}_{f}}+\sum_{w \in S}\left\langle g, \alpha_{w}\right\rangle_{w}\left(e_{w}-\alpha_{w}\right)$. By Lemma 2.1, it suffices to show that if $S \neq S^{\prime}$ then $g_{S}$ and $g_{S^{\prime}}$ have distinct images. To see this, let $v \in S \backslash S^{\prime}$ and let $C$ be the direct sum of $X$ and $\sum_{w \neq v} \mathbb{F} e_{w}$. Then the image of $g_{S^{\prime}}$ lie in $C$ but that of $g_{S}$ doesn't lie in $C$ (the functional $\left\langle g, \alpha_{v}\right\rangle_{v}$ is nonzero because $\operatorname{im}(g)=\operatorname{im}(f)$ ). This completes the proof.

Proof of Theorem 2.5. Since $M \neq 0$ there exists an $n$ such that $M\left(\mathbb{F}^{n}\right) \neq 0$, and so it suffices to prove the second assertion. By Proposition 2.3, it suffices to show that $M_{n, \Theta} \subset M$. In other words, it suffices to show that if $\theta \in \Theta$ is arbitrary, then $L_{\mathcal{V}_{f_{n}}}\left(\left[f_{n}^{\mathcal{E}_{f_{n}}}\right] \otimes \theta\right) \in M$.

Let $x \in M\left(\mathbb{F}^{n}\right)$ be a nonzero element. For every subspace $W$ of $\mathbb{F}^{n}$, choose a VI-morphism $f_{W}: \mathbb{F}^{d} \rightarrow \mathbb{F}^{n}$. We assume that the natural inclusion $f_{n}: \mathbb{F}^{d} \rightarrow \mathbb{F}^{n}$ is among these choices of morphisms. Now every element of $\mathcal{J}(\Theta)\left(\mathbb{F}^{n}\right)$ can be written uniquely as $\sum_{W}\left[f_{W}\right] \otimes \theta_{W}$ where $\theta_{W} \in \Theta$. In particular, we have $x=\sum_{W}\left[f_{W}\right] \otimes \theta_{W}$. Since $x \neq 0$ there exists a $W_{0}$ such that $\theta_{W_{0}} \neq 0$.

Now let $\theta$ be an arbitrary element of $\Theta$. Since $\Theta$ is irreducible, we can write $\theta$ as

$$
\theta=\sum_{\sigma \in \mathbf{G L}_{d}} a_{\sigma} \sigma \theta_{W_{0}}
$$

where $a_{\sigma} \in \mathbf{k}$. For $\sigma \in \mathbf{G L}_{d}$, let $\tau_{\sigma} \in \mathbf{G L}_{n}$ be an automorphism such that $\tau_{\sigma} \circ f_{W_{0}}=f_{n} \circ \sigma$. Such an automorphism exists by transitivity of action of $\mathbf{G L}_{n}$ on $d$-dimensional subspaces of $\mathbb{F}^{n}$. It is easy to see that $\operatorname{im}\left(\tau_{\sigma} f_{W}\right)=\operatorname{im}\left(f_{n}\right)$ if and only if $W=W_{0}$. Set $y=\sum_{\sigma \in \mathbf{G L}_{d}} a_{\sigma} \tau_{\sigma} x$. Clearly, $y \in M\left(\mathbb{F}^{n}\right)$. We have

$$
\sum_{\sigma \in \mathbf{G L}_{d}} a_{\sigma} \tau_{\sigma}\left[f_{W_{0}}\right] \otimes \theta_{W_{0}}=\sum_{\sigma \in \mathbf{G L}_{d}} a_{\sigma}\left[f_{n} \sigma\right] \otimes \theta_{W_{0}}=\left[f_{n}\right] \otimes\left(\sum_{\sigma \in \mathbf{G L}_{d}} a_{\sigma} \sigma \theta_{W_{0}}\right)=\left[f_{n}\right] \otimes \theta .
$$

This shows that $y$ can be written as

$$
y=\left[f_{n}\right] \otimes \theta+\sum_{W \neq \operatorname{im}\left(f_{n}\right)}\left[f_{W}\right] \otimes \theta_{W}^{\prime}
$$

for some $\theta_{W}^{\prime} \in \Theta$. Now let $\ell: \mathbb{F}^{n} \rightarrow \mathbb{F}^{n}+\mathcal{E}_{f_{n}}$ be the natural inclusion. Since $y \in M\left(\mathbb{F}^{n}\right)$, we conclude that $L_{\mathcal{V}_{n}}\left(\ell_{\star}(y)\right) \in M\left(\mathbb{F}^{n}+\varepsilon_{f_{n}}\right)$. We have

$$
\ell_{\star}(y)=\left[f_{n}^{\mathcal{E}_{f_{n}}}\right] \otimes \theta+\sum_{W \neq \mathrm{im}\left(f_{n}\right)}\left[f_{W}^{\mathcal{E}_{f_{n}}}\right] \otimes \theta_{W}^{\prime} .
$$


By Lemma 2.6, we conclude that

$$
L \mathcal{V}_{f_{n}}\left(\ell_{\star}(y)\right)=L \mathcal{V}_{f_{n}}\left(\left[f_{n}^{\varepsilon_{f_{n}}}\right] \otimes \theta\right)+\sum_{W \neq \operatorname{im}\left(f_{n}\right)} L \mathcal{V}_{f_{n}}\left(\left[f_{W}^{\mathcal{E}_{f_{n}}}\right] \otimes \theta_{W}^{\prime}\right)=L \mathcal{V}_{f_{n}}\left(\left[f_{n}^{\varepsilon_{f_{n}}}\right] \otimes \theta\right) \in M\left(\mathbb{F}^{n}+\mathcal{E}_{f_{n}}\right)
$$

This proves that $M_{n, \Theta} \subset M$, finishing the proof.

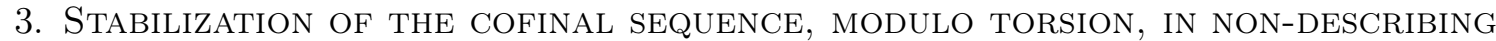 CHARACTERISTIC}

In this subsection, we assume that $q=|\mathbb{F}|$ is invertible in $\mathbf{k}$.

Let $f: \mathbb{F}^{d} \rightarrow X$ be a morphism in VI. Let $W$ be the image of $f$ with ordered basis $\left(\mathcal{B}_{f}, \prec\right)$, and $g: \mathbb{F}^{d} \rightarrow W$ be the restriction of $f$ to $W$. Let $<_{\alpha}$ be a linear order on complements of the line $\mathbb{F} \alpha$ in $W$ that contain $\left(\mathcal{B}_{f}\right)_{\prec \alpha}$, and $<^{\prime}$ be a linear order on complements of $W$ in $V$. Let $<$ be the lexicographic order on $\mathcal{V}_{f}$, that is, to check $v<w$ we first compare $\alpha$ components and then $C$ components and at last the $C^{\prime}$ components. Order $\mathcal{V}_{g}$ using the same lexicographic order as above. There is a natural order preserving (non-strictly) projection map $\pi: \mathcal{V}_{f} \rightarrow \mathcal{V}_{g}$ and, for $x \in \mathcal{V}_{g}$, we denote the fiber of this map at $x$ by $\pi^{-1}(x)$. More explicitly $\pi$ is given by $\left(\alpha, C, C^{\prime}\right) \mapsto(\alpha, C, 0)$.

Let ${ }^{\wedge}: X+\varepsilon_{f} \rightarrow X+\mathcal{E}_{f}$ be the VI automorphism that takes $e_{v}$ to $\hat{e}_{v}:=e_{v}-\alpha_{v}$ for each $v$ and fixes $V$ pointwise. Let $\mathcal{U}_{x}$ be the unipotent subgroup (with respect to the order defined by $<$ ) of the automorphism group of $\hat{\mathcal{E}}_{x}:=\mathbb{F}\left[\left\{\hat{e}_{v}: v \in \pi^{-1}(x)\right\}\right]$, that is, $\sigma \in \mathcal{U}_{x}$ if and only if for each $v \in \pi^{-1}(x)$ the element $\sigma \hat{e}_{v}-\hat{e}_{v}$ is a linear combination of $\hat{e}_{w}$ with $v<w \in \pi^{-1}(x)$. In particular, $\mathcal{U}_{x}$ fixes

$\hat{e}_{\max \left(\pi^{-1}(x)\right)}$. Clearly, the size of $\mathcal{U}_{x}$ is a power of $q$. From now on, we regard $\mathcal{U}_{x}$ as a subgroup of $\mathbf{G L}\left(X+\mathcal{E}_{f}\right)$ that fixes $X$ and $\hat{e}_{w}$ pointwise for each $w \notin \pi^{-1}(x)$.

Lemma 3.1. If $x \neq y$ then $\mathfrak{U}_{x} \cap \mathcal{U}_{y}$ and $\left[\mathfrak{U}_{x}, \mathcal{U}_{y}\right]$ are trivial. In particular, $\mathfrak{U}_{x} \mathfrak{U}_{y}=\mathfrak{U}_{x} \times \mathcal{U}_{y}$.

Proof. This is immediate.

For an initial segment $I$ of $\mathcal{V}_{g}$ we define $\mathcal{U}_{I}$ to be the subgroup $\prod_{x \in I} \mathcal{U}_{x}$. For $x \in \mathcal{V}_{g}$, let $D_{x}$ be the singleton consisting of $\max \left(\pi^{-1}(x)\right.$ ) (where the max is taken with respect to $<$ on $\mathcal{V}_{f}$ ), and let $D_{I}$ be the union of $D_{x}$ for $x \in I$.

Lemma 3.2. Let $x \in \mathcal{V}_{g}$ and let $X$ be a subset of $\mathcal{V}_{f}$ contained in $\sqcup_{y<x} \pi^{-1}(y)$. Then we have

$$
\frac{1}{\left|\mathcal{U}_{x}\right|} \sum_{\sigma \in \mathcal{U}_{x}} \sigma L_{\pi^{-1}(x)} L_{X}\left(\left[f^{\mathcal{E}_{f}}\right]\right)=L_{D_{x}} L_{X}\left(\left[f^{\mathcal{E}_{f}}\right]\right) .
$$

Proof. Let $g_{X^{\prime}}=f^{\mathcal{E}_{f}}+\sum_{v \in X^{\prime} \subset X}\left\langle f, \alpha_{v}\right\rangle_{v} \hat{e}_{v}$. By Lemma 2.1, $L_{X}\left(\left[f^{\mathcal{E}_{f}}\right]\right)$ is a linear combination of elements of the form $\left[g_{X^{\prime}}\right]$. So let $g=g_{X^{\prime}}$ for some subset $X^{\prime}$ of $X$. By linearity, it suffices to show that $\frac{1}{\left|\mathcal{U}_{x}\right|} \sum_{\sigma \in \mathcal{U}_{x}} \sigma L_{\pi^{-1}(x)}([g])=L_{D_{x}}([g])$. Note that $\sigma g=g$ for each $\sigma \in \mathcal{U}_{x}$. Thus by Lemma 2.1, 
we have

$$
\begin{aligned}
\sum_{\sigma \in \mathfrak{U}_{x}} \sigma L_{\pi^{-1}(x)}([g]) & =\sum_{\sigma \in \mathfrak{U}_{x}} \sigma\left(\sum_{S^{\prime} \subset \pi^{-1}(x)}(-1)^{\left|S^{\prime}\right|}\left[g+\sum_{v \in S^{\prime}}\left\langle f, \alpha_{v}\right\rangle_{v} \hat{e}_{v}\right]\right) \\
& =\sum_{\sigma \in \mathfrak{U}_{x}}\left(\sum_{S^{\prime} \subset \pi^{-1}(x)}(-1)^{\left|S^{\prime}\right|}\left[g+\sum_{v \in S^{\prime}}\left\langle f, x_{v}\right\rangle_{v}\left(\sigma \hat{e}_{v}\right)\right]\right) \\
& =\sum_{S^{\prime} \subset \pi^{-1}(x)}(-1)^{\left|S^{\prime}\right|}\left(\sum_{\sigma \in \mathfrak{U}_{x}}\left[g+\sum_{v \in S^{\prime}}\left\langle f, \alpha_{v}\right\rangle_{v}\left(\sigma \hat{e}_{v}\right)\right]\right) \\
& =\sum_{S^{\prime} \subset \pi^{-1}(x)}(-1)^{\left|S^{\prime}\right|} \Phi\left(S^{\prime}\right)
\end{aligned}
$$

where $\Phi\left(S^{\prime}\right)$ is the expression in big round brackets in the fourth expression. Suppose $S^{\prime} \subset \pi^{-1}(x)$ is nonempty and does not contain $D_{x}$, and let $S^{\prime \prime}=S^{\prime} \sqcup D_{x}$. We claim that $\Phi\left(S^{\prime}\right)=\Phi\left(S^{\prime \prime}\right)$. To see this, first note that the functional $\left\langle f, \alpha_{v}\right\rangle_{v}$ does not depend on $v \in \pi^{-1}(x)$ because $\left(\alpha_{v}, C_{v}\right)=\left(\alpha_{w}, C_{w}\right)$ for $v, w \in \pi^{-1}(x)$. Now for each $\sigma \in \mathcal{U}_{x}$ given by $\sigma\left(\hat{e}_{v}\right)=e_{v}+\sum_{w>v} a_{v, w} \hat{e}_{w}$, define $\tau \in \mathcal{U}_{x}$ by $\tau\left(\hat{e}_{v}\right)=e_{v}+\sum_{w>v} b_{v, w} \hat{e}_{w}$ where

$$
b_{v, w}= \begin{cases}a_{v, w}-1 & \text { if } w \in D_{x} \text { and } v=\min S^{\prime} \\ a_{v, w} & \text { otherwise. }\end{cases}
$$

By construction, we have

$$
\sum_{v \in S^{\prime}} \sigma \hat{e}_{v}=\sum_{v \in S^{\prime \prime}} \tau \hat{e}_{v}
$$

Since the functional $\left\langle f, \alpha_{v}\right\rangle_{v}$ does not depend on $v \in \pi^{-1}(x)$, we see that $\left[g+\sum_{v \in S^{\prime}}\left\langle f, \alpha_{v}\right\rangle_{v}\left(\sigma \hat{e}_{v}\right)\right]=\left[g+\left\langle f, \alpha_{v}\right\rangle_{v} \sum_{v \in S^{\prime}}\left(\sigma \hat{e}_{v}\right)\right]=\left[g+\left\langle f, \alpha_{v}\right\rangle_{v} \sum_{v \in S^{\prime \prime}}\left(\tau \hat{e}_{v}\right)\right]=\left[g+\sum_{v \in S^{\prime \prime}}\left\langle f, \alpha_{v}\right\rangle_{v}\left(\tau \hat{e}_{v}\right)\right]$. It follows immediately that $\Phi\left(S^{\prime}\right)=\Phi\left(S^{\prime \prime}\right)$, establishing the claim. The claim implies that we have

$$
\sum_{\sigma \in \mathfrak{U}_{x}} \sigma L_{\pi^{-1}(x)}([g])=\Phi(\varnothing)-\Phi\left(D_{x}\right) .
$$

Moreover, we have $\Phi(\varnothing)=\left|\mathcal{U}_{x}\right|[g]$ and $\Phi\left(D_{x}\right)=\left|\mathcal{U}_{x}\right|\left[g+\left\langle f, \alpha_{v}\right\rangle_{v}\left(e_{v}-\alpha_{v}\right)\right]$ where $v=\max \left(\pi^{-1}(x)\right)$. This shows that

$$
\sum_{\sigma \in \mathcal{U}_{x}} \sigma L_{\pi^{-1}(x)}([g])=\left|\mathcal{U}_{x}\right| L_{D_{x}}([g])
$$

completing the proof.

Lemma 3.3. Let $\sigma^{\prime} \in \prod_{y<x} \mathcal{U}_{y}$. If $v \in \pi^{-1}(x)$ then $\sigma^{\prime}$ commutes with $\sigma_{v}$.

Proof. We have $V+\varepsilon_{f}=\left(\sum_{y<x} \hat{\varepsilon}_{y}\right)+V+\left(\sum_{x \leq y} \hat{\varepsilon}_{y}\right)$. Clearly, $\sigma^{\prime}$ fixes $V+\left(\sum_{x \leq y} \hat{\varepsilon}_{y}\right)$ pointwise and stabilizes $\sum_{y<x} \hat{\mathcal{E}}_{y}$. On the other hand, $\sigma_{v}$ fixes $\sum_{y<x} \hat{\mathcal{E}}_{y}$ pointwise and stabilizes $V+\left(\sum_{x \leq y} \hat{\mathcal{E}}_{y}\right)$. The assertion follows from this.

Lemma 3.4. We have $\frac{1}{\mid \mathcal{U}_{v_{g}}} \sum_{\sigma \in \mathfrak{U}_{v_{g}}} \sigma L_{\mathcal{V}_{f}}\left(\left[f^{\mathcal{E}_{f}}\right]\right)=L_{D_{v_{g}}}\left(\left[f^{\mathcal{E}_{f}}\right]\right)$. 
Proof. Let $I$ be an initial segment of $\mathcal{V}_{g}$. We prove by induction on the size of $I$ that

$$
\sum_{\sigma \in \mathcal{U}_{I}} \sigma L_{\pi^{-1}(I)}\left(\left[f^{\mathcal{E}_{f}}\right]\right)=\left|\mathcal{U}_{I}\right| L_{D_{I}}\left(\left[f^{\mathcal{E}_{f}}\right]\right)
$$

The $|I|=1$ case follows from Lemma 3.2. Next, suppose $I=X \sqcup\{x\}$ for some initial segment $X$ of $\mathcal{B}_{f}$. Then we have

$$
\begin{aligned}
& \sum_{\sigma \in \mathcal{U}_{I}} \sigma L_{\pi^{-1}(I)}\left(\left[f^{\mathcal{E}_{f}}\right]\right)=\sum_{\left(\sigma, \sigma^{\prime}\right) \in \mathcal{U}_{x} \times \mathcal{U}_{X}}\left(\sigma, \sigma^{\prime}\right) L_{\pi^{-1}(x)} L_{\pi^{-1}(X)}\left(\left[f^{\mathcal{E}_{f}}\right]\right) \\
& =\left(\sum_{\sigma \in \mathcal{U}_{x}} \sigma L_{\pi^{-1}(x)}\right)\left(\sum_{\sigma^{\prime} \in \mathcal{U}_{X}} \sigma^{\prime} L_{\pi^{-1}(X)}\right)\left(\left[f^{\mathcal{E}_{f}}\right]\right) \quad \text { by Lemma } 3.3 \\
& =\left|\mathcal{U}_{X}\right|\left(\sum_{\sigma \in \mathcal{U}_{x}} \sigma L_{\pi^{-1}(x)}\right) L_{D_{X}}\left(\left[f^{\mathcal{E}_{f}}\right]\right) \quad \text { by induction } \\
& =\left|\mathcal{U}_{X}\right|\left|\mathcal{U}_{x}\right| L_{D_{x}} L_{D_{X}}\left(\left[f^{\mathcal{E}_{f}}\right]\right) \\
& \text { by Lemma } 3.2 \\
& =\left|\mathcal{U}_{I}\right| L_{D_{I}}\left(\left[f^{\mathcal{E}_{f}}\right]\right)
\end{aligned}
$$

completing the proof.

We now recall and prove the following theorem from the introduction (copy of Theorem 1.8).

Theorem 3.5. Let $\Theta$ be any representation of $\mathbf{G L}_{d}$. Then the descending chain $M_{d, \Theta} \supset M_{d+1, \Theta} \supset$ ... stabilizes in $\operatorname{Mod}_{\mathrm{VI}}^{\mathrm{gen}}$. In fact, $M_{d, \Theta} / M_{n, \Theta}$ is supported in degrees $<n+q^{d(n-d)}\left(1+q+\cdots+q^{d-1}\right)$. In other words, $M_{n, \Theta}=M_{d, \Theta}$ in $\operatorname{Mod}_{\mathrm{VI}}^{\text {gen }}$ for each $n \geq d$.

Proof. Fix an $n \geq d$. Let $f: \mathbb{F}^{d} \rightarrow \mathbb{F}^{n}$ be the natural inclusion. Denote the image of $f$ by $W$, and the restriction $g: \mathbb{F}^{d} \rightarrow W \cong \mathbb{F}^{d}$ by $g$. Let $C^{\prime}$ be the maximal complement of $W$ with respect to the order $<^{\prime \prime}$ (as defined in the beginning of this subsection). By definition, $D_{\mathcal{V}_{g}}=\mathcal{V}_{g} \times\left\{C^{\prime}\right\}$. Let $\varphi: \nu_{g} \rightarrow D_{v_{g}}$ be the bijection taking $(\alpha, C, 0)$ to $\left(\alpha, C, C^{\prime}\right)$, and note that $D_{v_{g}}=\operatorname{im}(\varphi)$. The map $\varphi$ together with $f$ induces a map $\widetilde{\varphi}: \mathbb{F}^{d}+\mathcal{E}_{g} \rightarrow \mathbb{F}^{n}+\mathcal{E}_{f}$. Let $\theta \in \Theta$. Then we have

$$
\begin{aligned}
\widetilde{\varphi}_{\star}\left(L_{\mathcal{V}_{g}}\left(\left[g^{\mathcal{E}_{g}}\right] \otimes \theta\right)\right) & =L_{D_{v_{g}}}\left(\left[f^{\mathcal{E}_{f}}\right] \otimes \theta\right) \\
& =L_{D_{v_{g}}}\left(\left[f^{\mathcal{E}_{f}}\right]\right) \otimes \theta \\
& =\frac{1}{\left|\mathcal{U}_{v_{g}}\right|} \sum_{\sigma \in \mathcal{U}_{v_{g}}} \sigma L_{\mathcal{V}_{f}}\left(\left[f^{\mathcal{E}_{f}}\right]\right) \otimes \theta \\
& =\frac{1}{\left|\mathcal{U}_{\mathcal{V}_{g}}\right|} \sum_{\sigma \in \mathcal{U}_{v_{g}}} \sigma L_{\mathcal{V}_{f}}\left(\left[f^{\mathcal{E}_{f}}\right] \otimes \theta\right) \quad \text { by Lemma } 3.4 \\
& \in M_{n, \Theta} .
\end{aligned}
$$

Since $\theta$ is arbitrary, we conclude that $M_{d} / M_{n}$ is supported in degrees $<\operatorname{dim}_{\mathbb{F}}\left(\mathbb{F}^{n}+\mathcal{E}_{f}\right)$, and hence is torsion. Clearly, we have

$$
\operatorname{dim}_{\mathbb{F}}\left(\mathbb{F}^{n}+\varepsilon_{f}\right)=n+q^{d(n-d)}\left(1+q+\cdots+q^{d-1}\right) .
$$

This completes the proof.

Corollary 3.6. Let $\Theta$ be an irreducible representation of $\mathbf{G L}_{d}$. Then, in $\operatorname{Mod}_{\mathrm{VI}}^{\text {gen }}, M_{n, \Theta}$ is irreducible and is the socle of $\mathcal{J}(\Theta)$. 
Proof. This follows immediately from Theorem 2.5 and Theorem 3.5.

\section{Classification of irReducibles}

Throughout, in this section, we shall assume that $q$ is invertible in $\mathbf{k}$. We will now make heavy use of the first paper [Nag] in this sequel. We have kept all of its notation. In particular, we need the following notation:

- $\Gamma(M)$ is the maximal torsion submodule of $M$.

- $\mathbf{S}$ is the saturation functor and is given by the composition $\mathrm{S} \circ \mathrm{T}$ of section and localization functors from the introduction.

- $\Sigma, \bar{\Sigma}$ are two endofunctors on ModVI $_{\mathrm{VI}}$ called shift functors. Both of them commute with $\Gamma$; see [Nag, Proposition 4.27].

The following theorem summarizes some of the results from the first paper that we need:

Theorem 4.1 ([Nag]). Let $M$ be a finitely generated VI-module. Then we have the following

(a) There is a polynomial $P$ of such that for $n \gg 0$ we have $\operatorname{dim}_{\mathbf{k}} M\left(\mathbb{F}^{n}\right)=P\left(q^{n}\right)$. The invariant $\operatorname{deg} P$ is called the $\delta$-invariant and is denoted by $\delta(M)$. If $\Theta$ is a representation of $\mathbf{G L}_{d}$, then we have $\delta(\mathcal{J}(\Theta))=d$.

(b) For large enough $d, \Sigma^{d} M$ and $\bar{\Sigma}^{d} M$ are semi-induced. Moreover, $\delta(M)=\delta\left(\bar{\Sigma}^{d} M\right)=\delta\left(\Sigma^{d} M\right)$ for any $d$.

(c) There is an exact triangle

$$
\mathrm{R} \Gamma(M) \rightarrow M \rightarrow \mathrm{RS}(M) \rightarrow
$$

where $\mathrm{R} \Gamma(M)$ is represented by a finite complex of finitely generated torsion modules and $\operatorname{RS}(M)$ is represented by a finite complex of finitely generated induced modules with $\delta$-invariant at most $\delta(M)$.

(d) If $T$ is torsion and $I$ is induced then $\operatorname{RHom}(T, I)=0$.

(e) Let $\Theta$ be a representation of $\mathbf{G L}_{d}$. Suppose $I$ is an induced module with $\delta(I)<d$. Then $\operatorname{RHom}(I, \mathcal{J}(\Theta))=0$.

(f) We have $\delta(\mathbf{S}(M))=\delta(M)$.

Here is an immediate corollary.

Corollary 4.2. Let $M$ be a finitely generated VI-module with $\delta(M)<0$, and let $\Theta$ be a representation of $\mathbf{G L}_{d}$. Then $\operatorname{RHom}(M, \mathcal{J}(\Theta))=0$.

Proposition 4.3. Let $\Theta$ be an irreducible representation of $\mathbf{G L}_{d}$. Suppose $M \subset \mathcal{J}(\Theta)$ is any nonzero submodule. Then $\delta(M)=d$.

Proof. Suppose, if possible, $\delta(M)<d$. The previous corollary implies that $\operatorname{RHom}(M, \mathcal{J}(\Theta))=0$, a contradiction because $\operatorname{Hom}(M, \mathcal{J}(\Theta)) \neq 0$.

Proposition 4.4. Let $\Theta$ be an irreducible representation of $\mathbf{G L}_{d}$. Suppose $M \subset \mathcal{J}(\Theta)$ is any nonzero submodule. Then $\delta(\mathcal{J}(\Theta) / M)<d$.

Proof. By Theorem 4.1(b), there is a $d$ such that $\bar{\Sigma}^{d} M$ is semi-induced. By Theorem 4.1(b) and the previous proposition, we have $\delta\left(\bar{\Sigma}^{d} M\right)=d$. Since $\bar{\Sigma}^{d} M$ is a submodule of $\bar{\Sigma}^{d} \mathcal{J}(\Theta)$, we see that the top part of $\bar{\Sigma}^{d} M$ must be $\mathcal{J}(\Theta)$. Since $\delta\left(\left(\bar{\Sigma}^{d} \mathcal{J}(\Theta)\right) / \mathcal{J}(\Theta)\right)<d$, we conclude that $\delta\left(\bar{\Sigma}^{d}(\mathcal{J}(\Theta) / M)\right)<d$. The result follows from Theorem 4.1(b). 
By part (e) of Theorem 4.1, we see that the $\delta$-invariant on VI-modules descends to the category of generic VI-modules. In other words, for a generic VI-module $N$, we can define $\delta(N)=\delta(\mathrm{S}(N))$. Then for any finitely generated VI-module $M$, we have $\delta(\mathrm{T}(M))=\delta(\mathbf{S}(M))=\delta(M)$.

Theorem 4.5. Set $\mathrm{L}(\Theta)=\mathrm{T}\left(M_{d, \Theta}\right)$. We have the following:

(a) Let $\Theta$ be an irreducible representation of $\mathbf{G L}_{d}$. Then $\mathrm{L}(\Theta)$ is irreducible and is the socle of $\mathrm{T}(\mathrm{J}(\Theta))$. Moreover, $\mathrm{T}(\mathrm{J}(\Theta))$ is of finite length.

(b) $\operatorname{Mod}_{\mathrm{VI}}^{\text {gen }}$ is artinian.

(c) The correspondence

$$
\bigsqcup_{n \geq 0} \operatorname{Irr}\left(\operatorname{Mod}_{\mathbf{k}\left[\mathbf{G L}_{n}\right]}\right) \rightarrow \operatorname{Irr}\left(\operatorname{Mod}_{\mathrm{VI}}^{\text {gen }}\right)
$$

given by $\Theta \mapsto \mathrm{L}(\Theta)$ is one-to-one.

Proof. Proof of (a). By Corollary 3.6, we see that $\mathrm{L}(\Theta)$ is irreducible and is the socle of $\mathrm{T}(\mathrm{J}(\Theta))$. For the second assertion, we proceed by induction on $d$. By the previous proposition, there is an $m \gg 0$ such that $\bar{\Sigma}^{m}\left(\mathcal{J}(\Theta) / M_{d, \Theta}\right)$ admits a filtration by induced modules of the form $\mathcal{J}(Z)$ where $Z$ is a $\mathbf{G L}_{k}$ representation for some $k<d$. By induction, $\mathrm{T}(\mathcal{J}(Z))$ is of finite length. The same must hold for $\mathrm{T}\left(\bar{\Sigma}^{m}\left(\mathcal{J}(\Theta) / M_{d, \Theta}\right)\right)$. Since $\mathrm{T}(\mathcal{J}(\Theta)) / \mathrm{L}(\Theta)$ embeds into $\mathrm{T}\left(\bar{\Sigma}^{m}\left(\mathcal{J}(\Theta) / M_{d, \Theta}\right)\right)$, we conclude that $\mathrm{T}(\mathcal{J}(\Theta)) / \mathrm{L}(\Theta)$ is of finite length. Thus (a) holds.

Proof of (b). Let $M$ be a finitely generated VI-module. Since $\mathrm{T}(M)$ embeds into $\mathrm{T}\left(\bar{\Sigma}^{m} M\right)$ it suffices to show that $\mathrm{T}\left(\bar{\Sigma}^{m} M\right)$ is of finite length for some $m$. Let $m \gg 0$ be such that $\bar{\Sigma}^{m} M$ is semi-induced. By part (a), T $\left(\bar{\Sigma}^{m} M\right)$ is of finite length for such an $m$. This shows that (b) holds.

Proof of (c). Let $M$ be a finitely generated VI-module such that $\mathrm{T}(M)$ is irreducible. We may assume that $M$ is torsion free. Let $m \gg 0$ be such that $\bar{\Sigma}^{m} M$ is semi-induced. Let

$$
0=M^{0} \subset \ldots \subset M^{r}=\bar{\Sigma}^{m} M
$$

be a filtration such that $M^{i} / M^{i-1} \cong \mathcal{J}\left(\Theta_{i}\right)$ for some $\mathbf{G L}_{i}$ representation $\Theta_{i}$. Since $M$ is torsion-free it embeds into $\bar{\Sigma}^{m} M$. Pick the least $i$ such that $M \cap M^{i}$ is nonzero. By irreducibility of $\mathrm{T}(M)$, the module $M /\left(M \cap M^{i}\right)$ is torsion. Also, $M \cap M^{i}$ embeds into $\mathcal{J}\left(\Theta_{i}\right)$. Thus $\mathrm{T}(M)=\mathrm{T}\left(M \cap M^{i}\right)$ must be the socle $\mathrm{L}\left(\Theta_{i}\right)$ of $\mathrm{T}\left(\mathcal{J}\left(\Theta_{i}\right)\right)$. Thus the correspondence $\Theta \mapsto \mathrm{L}(\Theta)$ is surjective.

To see injectivity, first note that if $\mathrm{L}\left(\Theta_{1}\right)$ and $\mathrm{L}\left(\Theta_{2}\right)$ are isomorphic then their $\delta$-invariants must be equal. Assume that it is $d$. Let $f: \mathrm{L}\left(\Theta_{1}\right) \rightarrow \mathrm{L}\left(\Theta_{2}\right)$ be an isomorphism. By definition of the Serre quotient, this map is induced by a map $g: A \rightarrow B$ where $A \subset M_{d, \Theta_{1}}$ and $B \subset M_{d, \Theta_{2}}$ are submodules satisfying $B, M_{d, \Theta_{1}} / A \in \operatorname{Mod}_{\mathrm{VI}}^{\text {tors }}$. The latter two modules are supported in finitely many degrees. Thus if $m$ is large enough, we see that $\bar{\Sigma}^{m} g: \bar{\Sigma}^{m} M_{d, \Theta_{1}} \rightarrow \bar{\Sigma}^{m} M_{d, \Theta_{2}}$ is an isomorphism. Further, we can assume that $m$ is large enough so that $\bar{\Sigma}^{m} M_{d, \Theta_{1}}$ and $\bar{\Sigma}^{m} M_{d, \Theta_{2}}$ are semi-induced. The top parts of these semi-induced modules are $\mathcal{J}\left(\Theta_{1}\right)$ and $\mathcal{J}\left(\Theta_{2}\right)$ respectively (the same argument as in the proof of the previous proposition holds). Since $\bar{\Sigma}^{m} g$ is an isomorphism, we see that it induces an isomorphism $\mathcal{J}\left(\Theta_{1}\right) \rightarrow \mathcal{J}\left(\Theta_{2}\right)$. This implies that $\Theta_{1}$ and $\Theta_{2}$ are isomorphic. This completes the proof of (c).

For a VI-module $M$, let $\mathcal{K}_{d}(M)$ denote the intersection of kernels of maps from $M$ to VI-modules generated in degrees $<d$. In other words, we have

$$
\mathcal{K}_{d}(M)=\bigcap_{\substack{f: M \rightarrow N \\ t_{0}(N)<d}} \operatorname{ker} f .
$$


Similarly, we define $\mathcal{K}_{d}^{\text {sat }}(M)$ to be the intersection of kernels of maps from $M$ to semi-induces VI-modules generated in degrees $<d$.

Lemma 4.6. The shift $\Sigma^{m}$ commutes with $\mathcal{K}_{d}$ and $\mathcal{K}_{d}^{\text {sat }}$ in the category of generic VI-modules, that is, we have the following:

(a) $\mathrm{T} \Sigma^{m} \mathcal{K}_{d}(M)=\mathrm{T} \mathcal{K}_{d} \Sigma^{m}(M)$.

(b) $\mathrm{T} \Sigma^{m} \mathcal{K}_{d}^{\mathrm{sat}}(M)=\mathrm{T} \mathcal{K}_{d}^{\mathrm{sat}} \Sigma^{m}(M)$.

Proof. Proof of (a). We first show that $\mathcal{K}_{d} \Sigma^{m}(M) \subset \Sigma^{m} \mathcal{K}_{d}(M) \subset \Sigma^{m} M$. Suppose $x \in \mathcal{K}_{d} \Sigma^{m}(M)$ and let $g: M \rightarrow N$ be a map with $t_{0}(M)<d$. Since $t_{0}\left(\Sigma^{m} N\right)<d$ and $x \in \mathcal{K}_{d} \Sigma^{m}(M)$, we see that $x \in \Sigma^{m}$ ker $g$. Thus we have

$$
\begin{aligned}
x \in \bigcap_{\substack{g: M \rightarrow N \\
t_{0}(N)<d}} \Sigma^{m} \operatorname{ker} g \\
=\Sigma^{m} \bigcap_{\substack{g: M \rightarrow N \\
t_{0}(N)<d}} \operatorname{ker} g \\
=\Sigma^{m} \mathcal{K}_{d}(M),
\end{aligned}
$$

proving our claim.

Let $\iota: M \rightarrow \Sigma^{m} M$ be the map induced by the VI-morphism $0 \rightarrow \mathbb{F}^{m}$. Suppose $x \in \Sigma^{m} \mathcal{K}_{d}(M)$. Let $N$ be a VI-module generated in degrees $<d$, and let $g: \Sigma^{m} M \rightarrow N$ be arbitrary. Then we have $\Sigma^{m}(g \circ \iota)(x)=0$ (note that $\left.\Sigma^{m}(g \circ \iota): \Sigma^{m} M \rightarrow \Sigma^{m} N\right)$. But $\Sigma^{m}(g \circ \iota)(x)=\Sigma^{m}(g) \circ \Sigma^{m}(\iota)(x)$. Since $g$ is arbitrary, we see that $\Sigma^{m}(\iota)(x) \in \Sigma^{m} \mathcal{K}_{d} \Sigma^{m} M$. This implies $\Sigma^{m}\left(\frac{\Sigma^{m} \mathcal{K}_{d}(M)}{\mathcal{K}_{d}\left(\Sigma^{m} M\right)}\right)=0$ and so $\frac{\Sigma^{m} \mathcal{K}_{d}(M)}{\mathcal{K}_{d}\left(\Sigma^{m} M\right)}$ is torsion completing the proof.

Proof of (b). The same proof as in Part (a) works as semi-induced modules generated in degrees $<d$ are closed under shift.

Theorem 4.7. Suppose $\Theta$ is an irreducible $\mathbf{G L}_{d}$ representation. Then we have

$$
\mathrm{T}\left(\mathcal{K}_{d}(\mathcal{J}(\Theta))\right)=\mathrm{T}\left(\mathcal{K}_{d}^{\mathrm{sat}}(\mathcal{J}(\Theta))\right)=\mathrm{L}(\Theta)
$$

where $\mathrm{L}(\Theta)=\mathrm{T}\left(M_{d, \Theta}\right)$, as in the previous theorem. Moreover, we have the following:

(a) $\mathrm{S}(\mathrm{L}(\Theta))=\mathcal{K}_{d}^{\mathrm{sat}}(\mathcal{J}(\Theta))$. In other words, $\mathcal{K}_{d}^{\mathrm{sat}}(\mathcal{J}(\Theta))$ is the saturation of $M_{d, \Theta}$.

(b) $\operatorname{deg} \mathrm{R}^{i} \Gamma\left(\mathcal{K}_{d}^{\mathrm{sat}}(\mathcal{J}(\Theta))\right) \leq 2 d-2(i-1)$ for $i \geq 2$. In particular, $\mathrm{R}^{i} \Gamma\left(\mathcal{K}_{d}^{\mathrm{sat}}(\mathcal{J}(\Theta))=0\right.$ for $i>d+1$.

(c) $\mathcal{K}_{d}^{\text {sat }}(\mathcal{J}(\Theta))$ is generated in degrees $\leq 2 d$. Moreover, $t_{i}\left(\mathcal{K}_{d}^{\text {sat }}(\mathcal{J}(\Theta))\right) \leq 2 d-i$ for $i \geq 0$.

Proof. It is clear that $\mathcal{K}_{d}(\mathcal{J}(\Theta)) \subset \mathcal{K}_{d}^{\text {sat }}(\mathcal{J}(\Theta))$. We first show that $M_{d, \Theta} \subset \mathcal{K}_{d}(\mathcal{J}(\Theta)$ ). To see this suppose $\varphi: \mathcal{J}(\Theta) \rightarrow N$ be a map where $N$ is some VI-module generated in degrees $<d$. Fix a VI-morphism $f: \mathbb{F}^{d} \rightarrow X$. Since $N$ is generated in degrees $<d$, there are $x_{1}, \ldots, x_{r} \in \sqcup_{k<d} N\left(\mathbb{F}^{k}\right)$ and $g_{1}, \ldots, g_{r} \in \sqcup_{k<d} \operatorname{Hom}_{\mathrm{VI}}\left(\mathbb{F}^{k}, X\right)$ such that

$$
\varphi([f] \otimes \theta)=\sum_{i=1}^{r} g_{i}\left(x_{i}\right) .
$$

Then we have

$$
\begin{aligned}
\varphi\left(L_{\mathcal{V}_{f}}\left(\left[f^{\mathcal{E}_{f}}\right] \otimes \theta\right)\right) & =L_{\mathcal{V}_{f}}\left(\varphi\left(\left[f^{\mathcal{E}_{f}}\right] \otimes \theta\right)\right) \\
& =L_{\mathcal{V}_{f}}\left(\sum_{i=1}^{r} g_{i}^{\mathcal{E}_{f}}\left(x_{i}\right)\right)
\end{aligned}
$$


This last expression vanishes because of Lemma 2.6 as $\operatorname{im}(f) \neq \operatorname{im}(g)$. Thus we have $M_{d, \Theta} \subset$ $\mathcal{K}_{d}(\mathcal{J}(\Theta)) \subset \mathcal{K}_{d}^{\mathrm{sat}}(\mathcal{J}(\Theta))$

We now show that $\mathrm{TK}_{d}^{\mathrm{sat}}(\mathcal{J}(\Theta))=\mathrm{L}(\Theta)$. It suffices to show that $\mathrm{T} \Sigma^{m}\left(\mathcal{K}_{d}^{\text {sat }}(\mathcal{J}(\Theta)) / M_{d, \Theta}\right)=0$ for large enough $m$. By the previous lemma, we have

$$
\mathrm{T} \Sigma^{m}\left(\mathcal{K}_{d}^{\text {sat }}(\mathcal{J}(\Theta)) / M_{d, \Theta}\right)=\mathrm{T} \Sigma^{m} \mathcal{K}_{d}^{\text {sat }}\left(\mathcal{J}(\Theta) / M_{d, \Theta}\right)=\mathrm{T}_{d}^{\text {sat }}\left(\Sigma^{m}\left(\mathcal{J}(\Theta) / M_{d, \Theta}\right)\right) .
$$

By Theorem 4.5(a), J $(\Theta) / \mathrm{L}(\Theta)$ has $\delta$-invariant $<d$. Thus by Theorem 4.1(b), we see that $\Sigma^{m}(\mathcal{J}(\Theta) / \mathrm{L}(\Theta))$ is a semi-induced module generated in degrees $<d$ for any large enough $m$. This implies that $\mathcal{K}_{d}^{\text {sat }}\left(\Sigma^{m}(\mathcal{J}(\Theta) / \mathrm{L}(\Theta))\right)=0$, completing the proof.

Proof of (a). Since $\mathrm{T}\left(\mathcal{K}_{d}^{\text {sat }}(\mathcal{J}(\Theta))\right)=\mathrm{L}(\Theta)$, it suffices to show that $\mathcal{K}_{d}^{\text {sat }}(\mathcal{J}(\Theta))$ is saturated. It is clearly torsion-free. Since $\mathcal{J}(\Theta)$ is derived saturated and $\mathcal{K}_{d}^{\text {sat }}(\mathcal{J}(\Theta))$ is a torsion-free submodule of it, it suffices to show that $\mathcal{J}(\Theta) / \mathcal{K}_{d}^{\text {sat }}(\mathcal{J}(\Theta)$ ) is torsion-free. But this is clear because by definition of $\mathcal{K}_{d}^{\text {sat }}, \mathcal{J}(\Theta) / \mathcal{K}_{d}^{\text {sat }}(\mathcal{J}(\Theta))$ is a submodule of a direct sum of semi-induced modules. This proves (a).

Proof of (b). It follows immediately from [Nag, Corollary 5.2] and [GL, Theorem 1.1(1)]. A more detailed argument in the FI-module case is provided in [CMNR, Theorem 2.10(4)].

Proof of (c). By parts (a) and (b), the quantity $r\left(\mathcal{K}_{d}^{\mathrm{sat}}(\mathcal{J}(\Theta))\right)$ as in [Nag, Theorem 5.13] is bounded by $2 d$. By Theorem 4.1(c), we see that $t_{0}\left(\operatorname{RS}\left(\mathcal{K}_{d}^{\mathrm{sat}}(\mathcal{J}(\Theta))\right)\right) \leq d$. Thus the proof of [Nag, Theorem 5.13] gives us $t_{i}\left(\mathcal{K}_{d}^{\mathrm{sat}}(\mathcal{J}(\Theta))\right) \leq 2 d-i$ for $i \geq 0$. This completes the proof.

Theorem 1.1 and Theorem 1.3 now follow by the previous two theorems after noting that $\mathcal{L}(\Theta)=$ $\mathcal{K}_{d}^{\mathrm{sat}}(\mathcal{J}(\Theta))$.

\section{REFERENCES}

[BEH] Daniel Barter, Inna Entova-Aizenbud, Thorsten Heidersdorf. Deligne categories and representations of the infinite symmetric group. arXiv:1706.03645.

[CE] Thomas Church, Jordan S. Ellenberg. Homology of FI-modules. Geom. Topol. 21 (2017), no. 4, $2373-2418$. arXiv: 1506.01022 .

[CEF] Thomas Church, Jordan S. Ellenberg, Benson Farb. FI-modules and stability for representations of symmetric groups. Duke Math. J. 164 (2015), no. 9, 1833-1910. arXiv:1204.4533.

[CMNR] Thomas Church, Jeremy Miller, Rohit Nagpal, Jens Reinhold. Linear and quadratic ranges in representation stability. Adv. Math. 333 (2018), 1-40. arXiv:1706.03845.

[CO] J. Comes, V. Ostrik. On DeligneâĂŹs category $\underline{\operatorname{Rep}}^{\mathrm{ab}}\left(S_{d}\right)$. Algebr Number Theory 8 (2014), No. 2, $473-496$.

[Del] P. Deligne. La categorie des representations du groupe symetrique $S_{t}$, lorsque t n'est pas un entier naturel. Algebraic groups and homogeneous spaces, Tata Institute of Fundamental Research, 209-273, Mumbai (2007).

[DM] P. Deligne, J.S. Milne, Tannakian Categories, Hodge Cycles, Motives, and Shimura Varieties. Lecture Notes in Math. 900 (1982), 101-228.

[EHS] I. Entova-Aizenbud, V. Hinich, V. Serganova. Deligne categories and the limit of categories $\operatorname{Rep}(\mathbf{G L}(m \mid n))$. Int. Math. Res. Not. IMRN (To appear).

[GL] Wee Liang Gan, Liping Li. Bounds on homological invariants of VI-modules. arXiv:1710.10223.

[GLX] Wee Liang Gan, Liping Li, Changchang Xi. An application of Nakayama functor in representation stability theory. arXiv: 1710.05493.

[GW] Wee Liang Gan, John Watterlond. A representation stability theorem for VI-modules. Algebr. Represent. Theor. 21 (2018), no. 1, 47-60. arXiv:1602.00654.

[Har1] Nate Harman. Stability and periodicity in the modular representation theory of symmetric groups. arXiv: 1509.06414

[Har2] Nate Harman. Virtual specht stability for FI-modules in positive characteristic. J. Algebra 488 (2017), $29-41$. arxiv1607.04271.

[Nag] Rohit Nagpal. VI-modules in non-describing characteristic, part I. arXiv:1709.07591.

[PS] Andrew Putman, Steven V Sam. Representation stability and finite linear groups. Duke Math. J. 166 (2017), no. 13, 2521-2598. arXiv:1408.3694. 
[SS1] Steven V Sam, Andrew Snowden. GL-equivariant modules over polynomial rings in infinitely many variables. Trans. Amer. Math. Soc. 368 (2016), 1097-1158. arXiv:1206.2233.

[SS5] Steven V Sam, Andrew Snowden. Gröbner methods for representations of combinatorial categories. J. Amer. Math. Soc. 30 (2017), 159-203. arXiv:1409.1670.

Department of Mathematics, University of Chicago, Chicago, IL

E-mail address: nagpal@math.uchicago.edu

URL: http://math.uchicago.edu/ nagpal/ 